\title{
Tubercular mastitis: An institutional experience from a tertiary care centre of northern India
}

\author{
Amit Gupta ${ }^{1}$, Lovenish Bains ${ }^{2}$, Deepshikha Yadav ${ }^{3}$, Prashant Durgapal', \\ Manish Kumar Agrawal ${ }^{5}$ \\ ${ }^{1}$ Associate Professor, ${ }^{3}$ Senior Resident, Department of General Surgery, All India Institute Of Medical Sciences, \\ Rishikesh, Uttarakhand, India, ${ }^{2}$ Assistant Professor, Department of General Surgery, MAMC \& LN Hospital, New Delhi \\ India, ${ }^{4}$ Assistant Professor, Department of Pathology, All India Institute Of Medical Sciences, Rishikesh, Uttarakhand, \\ India, ${ }^{5}$ Assistant Professor, Department of General Surgery, Dr BSA Medical College \& Hospital, New Delhi, India
}

Background: Tuberculous mastitis (TM) is a rare extra pulmonary presentation of tuberculosis. It may be problematic to distinguish from carcinoma breast, a condition with which it may coexist. Fine needle aspiration cytology (FNAC)/biopsy are indispensable for diagnosis and tuberculosis culture when positive may be very valuable to guide antimicrobial therapy. Aims and Objectives: To disseminate the message to the concerned expertise that it can present a diagnostic problem on radiological and microbiological investigations, and thus a high index of suspicion is needed. Material and Methods: 19 cases of tubercular mastitis between January 2012 to July 2016 were identified and included in the present study. Cytology and biopsy alongwith AFB stain was done for confirmation. Results: Age ranged between 23- 55 years (median 33 years). Axillary nodes were palpable in 9 (47.3\%) patients. Acid Fast Bacilli stain which was positive in only 3 patients. All the 19 patients were started on antitubercular treatment. Conclusion: This study highlights the importance of early diagnosis and aggressive medical and if required surgical management to cure this disease.

\section{INTRODUCTION}

Tubercular mastitis (TB) is a rare entity accounting for less than $1 \%$ of all diseases of the breast in the developed countries. ${ }^{1,2}$ Incidence in India has been reported to be $1-4.5 \% .{ }^{3}$ It may be a part of systemic disease or may be the only manifestation of tuberculosis. It occurs more commonly in females of reproductive age group and less common in prepubescent and elderly women. ${ }^{4}$ There is often diagnostic dilemma, clinically and radiologically as breast carcinoma and breast abscess may co-exist with tubercular mastitis. ${ }^{5}$ Fine needle aspiration may be inconclusive so diagnosis is based on high index of suspicion, Langerhans' cells in the granulomatous lesion and patients improvement of clinical signs and symptoms on antitubercular treatment. We here report our institutional experience of pattern of occurrence, clinical features, cytopathological diagnosis and management of tubercular mastitis in nineteen patients.

\section{MATERIAL AND METHODS}

19 cases of tubercular mastitis between January 2012 to July 2016 were identified and included in the present study. Diagnosis of tubercular mastitis was made on the basis of clinical history of breast lump, discharging sinus and or accompanying breast abscess. Constitutional symptoms were low grade fever, loss of weight and decreased appetite. All the patients were considered for Chest X ray, bilateral breast Ultrasonography (USG), Fine needle aspiration cytology (FNAC) from breast lump and biopsy was done if FNAC was inconclusive. The cytology and histopathology material was subjected to Acid Fast Bacilli stain. Ultrasonography abdomen was done to rule out 
any retroperitoneal lymphadenopathy. Once diagnosis was confirmed patients were started on anti tubercular treatment (AT'T). Response was evaluated on the basis of weight gain, increase in appetite, remission of fever, healing of sinus, abscess and resolution of radiological findings. Patients were considered for breast abscess drainage if it did not resolve on ATT treatment.

\section{RESULTS}

Nineteen patients of tubercular mastitis were evaluated in the present study. All the patients were females. Age ranged between 23- 55 years (median 33 years). Diabetes mellitus and or hypertension were comorbidities in $52.6 \%(10 / 19)$ patients. 57.8\% (11/19) patients had left breast involvement, $36.8 \%$ (7/19) right breast and only 1 had involvement of both breast. The clinical presentation was abscess involving whole of the breast in $26.3 \%(5 / 19)$ patients, $15.7 \%(3 / 19)$ had discharging sinus and 26.3\% (5/19) had palpable breast lump. $31.5 \%(6 / 19)$ patients had mastalgia as the presenting symptom.(Figure 1a,b)

Axillary nodes were palpable in 47.3\% (9/19) patients whereas $52.6 \%(10 / 19)$ patients had no axillary nodes at presentation (Graph 1).

$78.9 \%(15 / 19)$ patients had fever at presentation with $31.5 \%(6 / 19)$ patients having associated loss of appetite and weight loss. Chest $\mathrm{X}$ ray revealed cavitatory lesion in $47.3 \%(9 / 19)$ patients, 36.8\% (7/19) had pleural effusion and 3 had normal skiagram. USG bilateral breast reported cystic lesion in 57.8\% (11/19) and multilocular abscess 42.1\% (8/19) patients (Figure 2).

Cytological assessment was positive for granulomatous lesion in only 36.8\% (7/19) patients. Biopsy was positive in cases were FNAC was inconclusive (Figure 3).

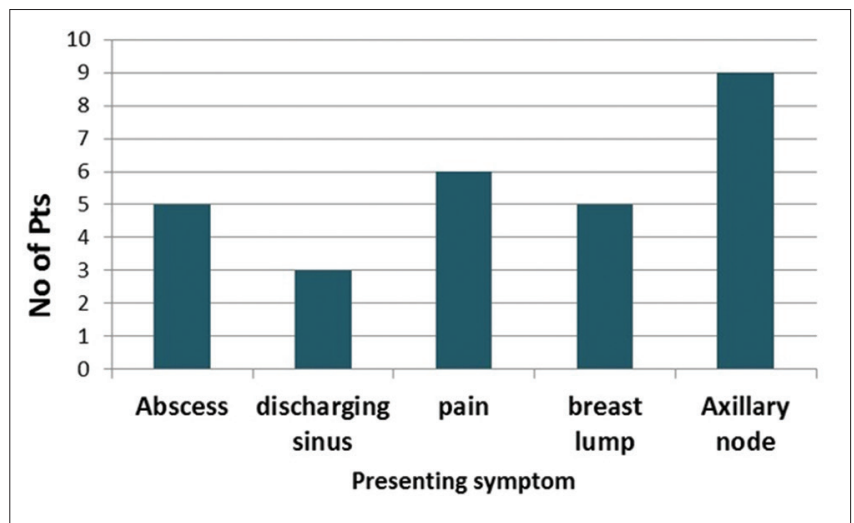

Graph 1: Showing presenting symptoms in patients of tubercular mastitis
FNAC and biopsy reported giant cell granulomas which was consistent with tuberculosis. In patients with palpable axillary nodes FNAC was done from node also. The cytology and histopathology material was subjected to Acid Fast Bacilli stain which was positive in only 3 patients.

All the 19 patients were started on antitubercular treatment. Breast abscess drainage was performed in 42.1\% (8/19) patients in view of patient being symptomatic. $5.26 \%(1 / 19)$

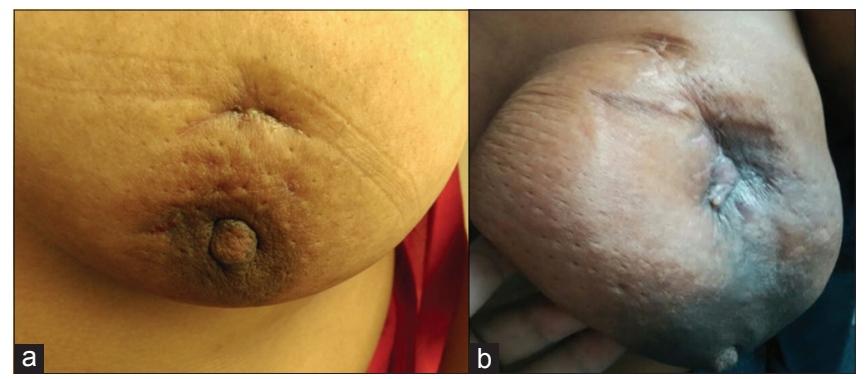

Figure 1: (a) showing discharging sinus just above the areolar region with puckering and peau d' orange appearance of the overlying skin (b) showing puckering of skin with underlying lump

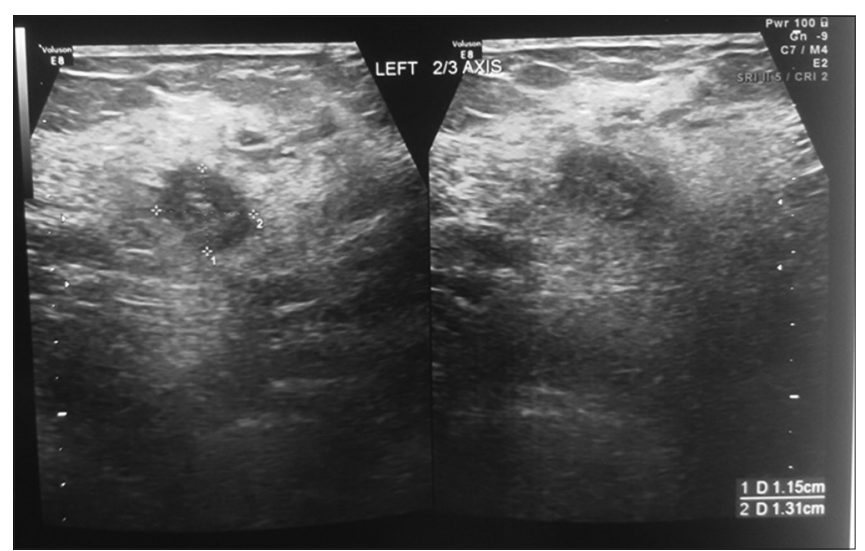

Figure 2: Ultrasonography left breast showing irregular marginated hypoechoic lesion 2-3 o clock position

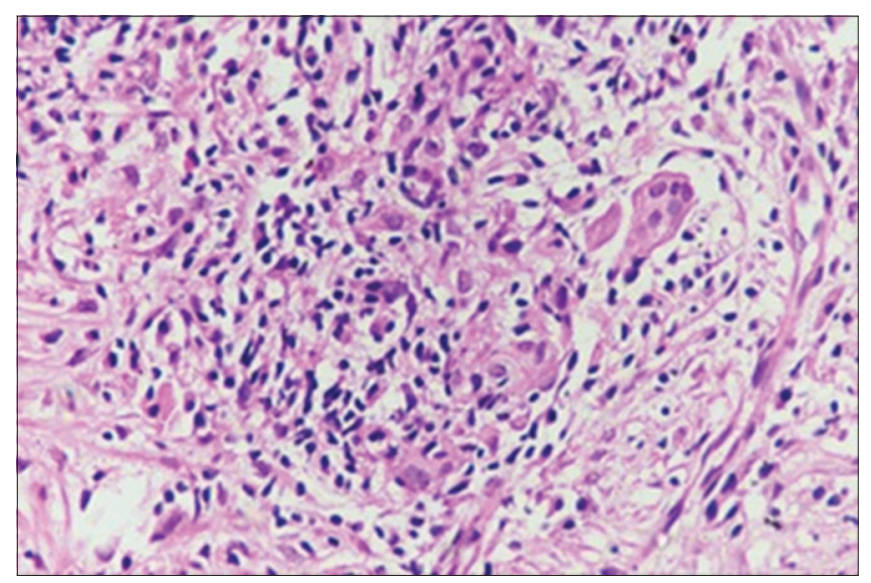

Figure 3: Hematoxylin and Eosin 40x stained section from breast lump shows giant cell granuloma consistent with tuberculosis 
patient had to be considered for simple mastectomy in view of non resolution of symptoms.

\section{DISCUSSION}

Primary and disseminated tuberculosis is a very common entity in India. Tubercular mastitis is a rare entity with primary tubercular mastitis being very rare. Tuberculosis of the breast was first documented in medical literature by Sir Astley Cooper in 1829. ${ }^{6}$ The incidence of breast tuberculosis amongst the total number of mammary conditions in several Indian series varies between 0.64 and 3.59 per cent. $^{7}$

Breast tissue is remarkably resistant to tuberculosis. Breast tuberculosis is considered to be primary when the breast lesion is the only manifestation of tuberculosis, and secondary when there is a demonstrable focus of tuberculosis elsewhere in the body as per Mckeown and Wilkinson classification. ${ }^{8}$ However, Vassilakos stated that primary breast tuberculosis was perhaps quite rare and was identified because the clinician was unable to notice the true focus of the disease. ${ }^{9}$ In our study only 3 patients had a normal Chest X-ray and did not have any other focus of tubercular infection with the probability of breast being the primary site of tuberculosis. The breast may become involved by haematogenous, lymphatic, spread from contiguous structures, direct inoculation, and ductal infection. Of these, the most putative opinion for spread of infection is centripetal lymphatic spread. ${ }^{10}$ As per Cooper's theory breast involvement is secondary to retrograde lymphatic extension. ${ }^{11}$ On the basis of this hypothesis axillary involvement may occur in 50$75 \%$ cases. In our study axillary lymphadenopathy was present in $47.3 \%(9 / 19)$ of patients. The history of the presenting symptoms in breast varies from few months to several years tuberculosis but is usually less than a year. It usually affects females in reproductive age group reason being frequent changes in the breast which predisposes it to trauma and infection. In this study also $57.8 \%$ $(11 / 19)$ females were in the reproductive age group. Breast tuberculosis is rare in males only in $4 \%$ cases and bilateral involvement is also very uncommon $(3 \%) \cdot{ }^{12}$ In the present study none of the patients were male and bilateral presentation was seen in only 1 patient. Breast tuberculosis most common presentation is a breast lump so its identity is mistaken with breast carcinoma. In our study only $26.3 \%(5 / 19)$ patients presented with breast lump with equally common presentation being breast abscess and pus discharging sinus was seen in 15.7\% (3/19) patients. Breast tuberculosis was initially classified into five different types by Mckeown and Wilkinson ${ }^{8}$ (i) Nodular tubercular mastitis, (ii) Disseminated or confluent tubercular mastitis, (iii) Sclerosing tubercular mastitis, (iv) Tuberculous mastitis obliterans, and (v) Acute miliary tubercular mastitis. But the new classification has omitted sclerosing tubercular mastitis and acute miliary tubercular mastitis as they are rarely encountered today. The nodular variant is often mistaken for a fibroadenoma or carcinoma and is the commonest accounting for $60 \%$ of cases. The disseminated variety commonly leads to caseation and sinus formation. Sclerosing TB affects older women and is slow growing with absence of suppuration. ${ }^{13}$

There is also much disagreement about the diagnostic norms for TB mastitis and various biopsies and FNA have been recommended. The gold standard diagnosis of TM is by bacteriological culture of breast tissue or by Ziehl Neelsen (ZN) stain. However, in TM the bacilli are isolated in only $25 \%$ of cases, and acid-fast bacilli (AFB) are identified only in $12 \%$ of the patients. Therefore, demonstration of caseating granulomas from the breast tissue and involved lymph nodes may be sufficient for the diagnosis. ${ }^{14}$ In our patients cytological assessment was positive for granulomatous lesion in only $36.8 \%(7 / 19)$ patients. FNAC and biopsy reported giant cell granulomas which was consistent with tuberculosis and Acid Fast Bacilli stain which was positive in only 15.7\% (3/19) of patients. Mantoux test is of no diagnostic value for breast tuberculosis and today stands obsolete. We also did not perform mantoux testing for our patients. The radiological investigations like mammography, computed tomography (CT-scan) and magnetic resonance imaging (MRI) of the breast have been extensively explored for the diagnosis of breast tuberculosis but of no benefit. Ultrasonography of the breast is cheap, easily accessible and helps in characterizing the lesion better (especially cystic from solid lesions) without exposure to radiation..$^{15}$

All patients should receive adequate anti tubercular therapy. Tuberculous mastitis should probably be treated as any other form of extra-pulmonary tuberculosis generally for six months (2HRZE/4HR) or nine months (2HRE/7HR, 2HRZ/7HR), unless drug resistance is present The first line drugs being ethambutol (E) $1200 \mathrm{mg}$; streptomycin (S) $750 \mathrm{mg}$, rifampicin (R) $450 \mathrm{mg}$, isoniazid $(\mathrm{H}) 600 \mathrm{mg}$ and pyrazinamide $(\mathrm{Z})$ $1500 \mathrm{mg}$. After complete ATT, residual lumps should be excised. Aspiration or surgical drainage may be required in some cases. In extensive cases and fulminant infection a simple mastectomy has been advocated. In our study one patient required simple mastectomy in view of fungating ulcerative lesion which persisted despite systemic therapy. Radical mastectomy is best avoided unless there is a co-existing malignancy. ${ }^{16,17}$ 


\section{CONCLUSION}

Tuberculosis of the breast is called the great mimicker. In the absence of well-defined clinical features, the true nature of the disease remains obscure and it is often mistaken for carcinoma or pyogenic breast abscess. It leads to diagnostic dilemma on radiological and cytopathological investigations, and thus a high index of suspicion is needed.

Incorporating a highly sensitive technique like PCR may be helpful in confirming the diagnosis early. AT'T remains the mainstay of treatment with surgery limited to aspiration of cold residual abscess and where coexisting malignancy needs to be ruled out. This study highlights the importance of early diagnosis and aggressive medical and if required surgical management to cure this disease.

\section{REFERENCES}

1. Harris SH, Khan MA, Khan R, Haque F, Syed A and Ansari MM Mammary Tuberculosis: Analysis of Thirty-Eight Patients. ANZ J Surg 2006; 76:234-237.

2. Kakkar S, Kapila K, Singh MK and Verma K. Tuberculosis of the Breast:ACytomorphologic Study. Acta Cytol 2000;44(3):292-296.

3. Aggarwal $\mathrm{V}$ and Bhargava P. Breast tuberculosis-a case report. J Indian Med Assoc 2008;106(1):38-40.

4. Jalali U, Rasul S, Khan A, Baig N, Khan A and Akhter R. Tuberculous Mastitis. J Coll Physicians Surg Pak 2005;15:234-237.
5. Alzaraa A and Dalal N. Coexistence of carcinoma and tuberculosis in one breast. World J Surg Oncol 2008; 6:29.

6. Cooper A. Illustration of the diseases of the breast. Part I. Longmans. Orme, Brown and Green. London: 1829: 73.

7. Dharkar RS, Kanhere MH, Vaishya ND and Baisarya AK. Tuberculosis of the breast. J Indian Med Assoc 1968; 50: 207-209.

8. Mckeown $\mathrm{KC}$ and Wilkinson $\mathrm{KW}$. Tuberculous diseases of the breast. Br J Surg 1952;39:420.

9. Vassilakos P. Tuberculosis of the breast: cytological findings with fine-needle aspiration. A case clinically and radiologically minicking carcinoma. Acta Cytol 1973; 17: 160-165.

10. Mukerjee P, George M, Maheshwari HB and Rao CP. Tuberculosis of the breast. J Indian Med Assoc 1974; 62: 410-412.

11. Domingo C, Ruiz J, Roig J, Texido A, Aguilar X and Morera J. Tuberculosis of the breast: a rare modern disease. Tubercle 1990; 71: 221-223.

12. Tewari $M$ and Shukla HS. Breast tuberculosis: diagnosis, clinical features \& management. Indian J Med Res 2005; 122:103-110.

13. Swarnagowri BN. Tuberculous Mastitis: A Case Report Sch J Med Case Rep 2013; 1(3):98-100.

14. Gupta D, Rajwanshi A, Gupta SK, Nijhawan R, Saran RK and Singh R. Fine Needle Aspiration Cytology in the Diagnosis of Tuberculous Mastitis. Acta Cytol 1999; 43:191-194.

15. Popli MB. Pictorial essay: tuberculosis of the breast. Indian J Radiol Imag 1999; 9: 127-132.

16. Banerjee SN, Ananthakrishnan N, Mehta RB and Parkash S. Tuberculous mastitis: a continuing problem. World J Surg 1987;11(1):105-109.

17. Al Soub $\mathrm{H}$ and Chacko K. Tuberculous mastitis: a rare disease. Br J Clin Pract 1996; 50(1):50-51.

\section{Authors Contribution:}

AG and LB-Study design; DY- Collection of Data; PD- Histopathological contribution; AG and MKG- Data analysis

Orcid ID:

Dr Amit Gupta: iD http://orcid.org/0000-0002-4847-4120

Dr Lovenish Bains:(1) http://orcid.org/0000-0002-8627-0452

Dr Deepshikha Yadav: http://orcid.org/0000-0002-9828-0666

Dr Prashant Durgapal:(i) http://orcid.org/0000-0002-8430-0216

Dr Manish Kumar Agrawal:(1) http://orcid.org/0000-0002-1456-2923

Source of Support: Nil, Conflict of Interest: Nil. 